\title{
Síndrome de sobrecarga en cuidadores familiares de adultos mayores y factores asociados. Chordeleg, 2017
}

\author{
The overload syndrome in family caregivers of elderly adults \\ and associated factors. Chordeleg, 2017 \\ Chamba Ortiz Paola Alexandra', Ojeda Orellana Karina Paola²
}

VOLUMEN 37 | No 1 | ABRIL 2019

FECHA DE RECEPCIÓN: 09/03/2019 FECHA DE APROBACIÓN: 17/04/2019 FECHA PUBLICACIÓN: $\quad 30 / 04 / 2019$

1. Libre ejercicio

2. Universidad de Cuenca

\begin{tabular}{c|l}
$\begin{array}{c}\text { Artículo } \\
\text { original }\end{array}$ & $\begin{array}{l}\text { Original } \\
\text { Article }\end{array}$
\end{tabular}

Correspondencia:

mdpaolacdagmail.com

Dirección:

Gualaceo, sector Ayaloma

Código Postal:

010350

Teléfono:

0998424390

Gualaceo - Ecuador

\section{RESUMEN}

Objetivo general: determinar la prevalencia del síndrome de sobrecarga en cuidadores familiares de adultos mayores y su relación con factores asociados en el cantón Chordeleg, durante el año 2017.

Material y métodos: es un estudio analítico transversal con 123 cuidadores familiares. Se utilizó el cuestionario del Instituto Nacional de Estadísticas y Censos, test de Zarit, índice de Katz y test FF-SIL. Las variables fueron expresadas en porcentajes y en frecuencias absolutas. Los factores asociados fueron evaluados a través de la razón de prevalencia con intervalo de confianza (95\%) y el Chi cuadrado.

Resultados: la mayor proporción de individuos tuvo 40 a 59 años de sexo femenino; el $73.17 \%$ son casados; el $40.65 \%$ tienen estudios primarios incompletos y en la relación con el paciente el $47.15 \%$ fueron hijos. El $58.50 \%$ de la población del estudio presentó sobrecarga, asociándose a tener más de 5 años como cuidador (RP 1.42 IC95\% 1.07-1.88, p=0.003) y a la incapacidad severa y moderada del paciente (RP 2.46, IC95\% 1.74 $-3.18, p=0.000$ ).

Conclusiones: el perfil del cuidador no difiere de otros estudios. Existió una alta prevalencia de síndrome de sobrecarga, asociándose a tener más de 5 años de cuidador y a la incapacidad moderada-severa del paciente.

Palabras claves: cuidadores, ancianos, estrés psicológico, afecciones crónicas múltiples. 


\section{ABSTRACT}

General objective: to determine the prevalence of Overload syndrome in family caregivers of older adults and its relationship with associated factors in Chordeleg canton during 2017.

Material and methods: it is a transversal analytical study with 123 family caregivers. The questionnaire of the National Institute of Statistics and Censuses, the Zarit test, the Katz index and the FF-SIL test were used. The variables were expressed in percentages and absolute frequencies. The associated factors were evaluated through the Prevalence Ratio with a confidence interval (95\%) and Chi square.

Results: the highest proportion of individuals was between 40 and 59 years of age; the $73.17 \%$ are married; $40.65 \%$ had incomplete primary studies and in the relationship with the patient, the $47.15 \%$ were children. The $58.50 \%$ of the study population showed overload, they have been associated with having more than 5 years as a caregiver (RR 1.42 IC95\% 1.07-1.88, $p=0.003$ ) and the severe and moderate disability of the patient (RP $2.46,95 \% \mathrm{Cl}$ 1.74-3.18, $p=0.000$ ).

Conclusions: the caregiver profile does not differ from other studies. There was a high prevalence of Overload syndrome, which is associated with having more than 5 years as a caregiver and the moderate-severe disability of the patient.

Keywords: caregivers, aged, stress, psychological, multiple chronic conditions.

\section{INTRODUCCION}

El cuidador informal es aquel individuo que brinda sus servicios de salud no profesionales a personas dependientes, generalmente sin percibir ninguna retribución económica, dentro de su domicilio y por lo general este rol puede desempeñarlo un miembro de la familia. Y brindan tres categorías de apoyo: apoyo instrumental (ayuda para realizar tareas o actividades de la vida diaria), apoyo informativo (consejo que se ofrece para solucionar problemas concretos) y apoyo emocional (expresión de sentimientos) [1].

Los adultos mayores que fueron considerados un segmento poblacional minoritario, son ahora el de más rápido crecimiento, lo que conlleva a la necesidad de más y mejores servicios de salud, para man- tener una adecuada calidad de vida que sostenga su independencia [2]. Por este motivo se requiere cuidados médicos, sociales y familiares de salud de larga duración [3].

Ampalam P.y cols. señalan múltiples consecuencias de la carga del cuidador como problemas de salud mental (depresión, ansiedad, estrés y síndrome de agotamiento), deterioro de la salud física (por ejemplo diabetes) y otros efectos negativos (disfunción familiar, aislamiento social, uso excesivo de servicios de salud y problemas financieros)[4].

Varios factores determinantes están asociados con la carga del cuidador, que pueden ser: factores relacionados con la enfermedad, clínicos, sociodemográficos, psicológicos y sociales [5]. Variables del paciente como: la enfermedad del paciente incluyen la edad [6] el grado de deterioro funcional y los síntomas neuro psiquiátricos [7]y las situaciones de crisis o problemas de comportamiento son relevantes [8].

Las variables que se relacionan con el cuidador incluyen: la edad avanzada [9], género femenino, ingreso familiar, grado de parentesco con el paciente [10] nivel de educación [11], presencia de comorbilidades físicas (hipertensión arterial [HTA] y diabetes mellitus) y/o mentales (depresión y ansiedad) [7] factores psicológicos sociales incluyen un bajo apoyo social y disfunción familiar [12]. Además, otras variables relacionadas con el aumento de la carga son la residencia y los días de contacto con el paciente [13].

\section{MATERIALES Y MÉTODOS}

Se realizó un estudio analítico de corte transversal. El Universo lo constituyeron 350 cuidadores familiares de adultos mayores que residen en el cantón Chordeleg, en el período de un año. La muestra fue probabilística y con selección aleatoria simple, su tamaño se calculó tomando en cuenta los grupos poblacionales específicos y considerando los factores asociados (se consideró el porcentaje del factor asociado con menor prevalencia equivalente al $12 \%$ ), mediante la fórmula indicada para estudios transversales con población conocida. El resultado estableció una muestra de 111 cuidadores y con un ajuste al $10 \%$ de pérdidas, la muestra final es de 123 participantes.

Criterios de inclusión: cuidador familiar de un adulto mayor que se encuentre en los registros del 
Centro de Salud de Chordeleg y Ministerio de Inclusión Económica y Social (MIES) Chordeleg y que acepte participar en la investigación con la firma del consentimiento informado. Criterios de exclusión: cuidadores menores de edad ( $<18$ años) y cuidadores profesionales.

La información se recolectó mediante la técnica de la entrevista estructurada, con un formulario diseñado por el autor, mismo que fue validado con un estudio piloto en el cantón Gualaceo de similares características sociodemográficas.

El nivel socioeconómico del hogar se evaluó mediante la encuesta del Instituto Nacional de Estadísticas y Censos (INEC) [14].

Para el grado de dependencia del adulto mayor se aplicó el índice de Katz, que consiste en la evaluación de seis funciones básicas que comprenden: baño, vestido, uso de retrete, movilidad, continencia y alimentación. La puntuación se la otorgó tomando en cuenta los siguientes valores: dependiente: 1 ; independiente 0 [15].

Se aplicó el test de sobrecarga del cuidador de Zarit, para lograr incluir diversas dimensiones del cuidador, consta de 22 preguntas de tipo Likert: $\sin$ sobrecarga $<47$, sobrecarga leve: $47-55$ e intensa: > 55 (50-52) [16]. La funcionalidad familiar se evaluó mediante el Test de FF-SIL que consta de 14 preguntas tipo liker que evalúan: cohesión, armonía, comunicación, adaptabilidad, roles, efectividad y permeabilidad [17].

Para caracterizar a la población se utilizó estadística descriptiva presentando en tablas simples con valores de frecuencia y porcentaje, medidas de tendencia central (media o mediana) y de dispersión (desviación estándar). Para determinar asociación entre la sobrecarga y sus factores asociados, se aplicó un análisis bivariado utilizando tablas de contingencia de $2 \times 2$, razón de prevalencia (RP), con intervalo de Confianza (IC) del 95\%, Chi Cuadrado, valor $p$, con significancia estadística $<0.05$, en caso de ser necesario se realiza el ajuste de Fisher.

Se procedió de acuerdo a los parámetros que contempla la declaración de Helsinki. Debido a que este proyecto involucra a seres humanos fue aprobada por el Comité de Bioética de la Facultad de Ciencias Médicas de la Universidad de Cuenca.

\section{RESULTADOS}

Con un total de 123 cuidadores el grupo de edad de 40 a 59 años representó el mayor porcentaje con el $36.59 \%$, seguido de los de 60 a 79 años $(27.64 \%)$. Las mujeres se encontraron en el $73.17 \%$. Se encontró además que el $73.17 \%$ están casados, seguido por los solteros $(20.33 \%)$. El $70.73 \%$ cursaron con la instrucción primaria, el $22.76 \%$ son analfabetas y hasta la secundaria llegó el $6.5 \%$. El $47.15 \%$ son hijos del adulto mayor y el $33.33 \%$ son su propia pareja (Tabla $\mathrm{N}^{\circ} 1$ ).

El $36.59 \%$ de adultos mayores se encuentra en el grupo etario mayor a 84 años y el sexo femenino representa el $58.54 \%$ (Tabla $\mathrm{N}^{\circ} 2$ ).

Se estableció que el $58,50 \%$ de los encuestados tuvo síndrome de sobrecarga (Tabla $\mathrm{N}^{\circ} 3$ ).

El $23.54 \%$ de cuidadores ejerció esta actividad por más de 5 años, (RP 1.42 IC95\% 1.07-1.88, $p=0.030$ ), de igual manera la dependencia con incapacidad severa y moderada del adulto mayor se presentó en el $46.34 \%$ de los casos (RP 2.46, IC $1.74-3.18, p=0.000)($ Tabla No 4$)$. 


\section{Tabla No 1}

Caracterización de cuidadores según edad, sexo, estado civil, escolaridad y relación del cuidador con el paciente. Chordeleg 2017

\begin{tabular}{|c|c|c|}
\hline & $n=123$ & $\%=100$ \\
\hline \multicolumn{3}{|l|}{ Edad } \\
\hline 20 a 39 años & 24 & 19.51 \\
\hline 40 a 59 años & 45 & 36.59 \\
\hline 60 a 79 años & 34 & 27.64 \\
\hline$>79$ años & 20 & 16.26 \\
\hline \multicolumn{3}{|l|}{ Sexo } \\
\hline Hombre & 33 & 26.83 \\
\hline Mujer & 90 & 73.17 \\
\hline \multicolumn{3}{|l|}{ Estado civil } \\
\hline Soltero & 25 & 20.33 \\
\hline Casado & 90 & 73.17 \\
\hline Unión Libre & 3 & 2.44 \\
\hline Viudo & 5 & 4.07 \\
\hline \multicolumn{3}{|l|}{ Escolaridad } \\
\hline Sin estudios & 28 & 22.76 \\
\hline Primaria incompleta & 50 & 40.65 \\
\hline Primaria completa & 37 & 30.08 \\
\hline Secundaria incompleta & 4 & 3.25 \\
\hline Secundaria completa & 4 & 3.25 \\
\hline \multicolumn{3}{|c|}{ Relación del cuidador con el paciente } \\
\hline Pareja & 41 & 33.33 \\
\hline Hijo/a & 58 & 47.15 \\
\hline Otro & 24 & 19.51 \\
\hline
\end{tabular}

*Media $=57,19 \pm 19,77$

Elaborado por: los autores. Fuente: base de datos. 
Tabla $N^{\circ} 2$

Caracterización de edad y sexo del adulto mayor.

\begin{tabular}{llcc}
\hline & & $\mathbf{n = 1 2 3}$ & $\%=100$ \\
\hline Edad & 65 a 74 años & 32 & 26.02 \\
& 75 a 84 años & 46 & 37.40 \\
& $>$ 84 años & 45 & 36.59 \\
\hline Sexo & & 51 & \\
& Hombre & & 41.46 \\
& & 72 & 58.54 \\
\hline
\end{tabular}

Elaborado por: los autores. Fuente: base de datos.

\section{Tabla No 3}

Prevalencia del síndrome de sobrecarga en cuidadores familiares de adultos mayores mediante el "Test de Zarit". Chordeleg 2017

\begin{tabular}{lll}
\hline Sobrecarga & $\mathbf{n = 1 2 3}$ & $\mathbf{\% = 1 0 0}$ \\
\hline $\mathbf{S i}^{*}$ & 72 & 58.50 \\
No & 51 & 41.5 \\
\hline
\end{tabular}

Elaborado por: los autores. Fuente: base de datos.

*Sobrecarga leve y sobrecarga intensa 


\section{Tabla N4}

Frecuencia del síndrome de sobrecarga en cuidadores familiares de adultos mayores y factores asociados. Chordeleg 2017

\begin{tabular}{|c|c|c|c|c|c|c|c|c|c|}
\hline & \multicolumn{4}{|c|}{ Sobrecarga } & \multirow{3}{*}{$\mathbf{R P}$} & \multirow{3}{*}{$\begin{array}{c}\text { IC } \\
95 \%\end{array}$} & \multirow{3}{*}{$\mathbf{p}$} \\
\hline & & & \multicolumn{2}{|c|}{ Si } & \multicolumn{2}{|c|}{ No } & & & \\
\hline & & & $n=72$ & $\%=58.54$ & $n=51$ & $\%=41.46$ & & & \\
\hline \multirow{2}{*}{\multicolumn{2}{|c|}{$\begin{array}{l}\text { Remuneración } \\
\text { económica }\end{array}$}} & No & 68 & 55.28 & 50 & 40.65 & \multirow{2}{*}{0.72} & $0.45-$ & \multirow{2}{*}{$0.402^{*}$} \\
\hline & & Si & 4 & 3.25 & 1 & 0.81 & & 1.14 & \\
\hline \multirow{2}{*}{\multicolumn{2}{|c|}{$\begin{array}{l}\text { Tiempo que lleva } \\
\text { como cuidador }\end{array}$}} & $>5$ años & 22 & 17.89 & 7 & 5.69 & \multirow{2}{*}{1.42} & $1.07-$ & \multirow{2}{*}{0.030} \\
\hline & & 0 a 5 años & 50 & 40.65 & 44 & 35.77 & & 1.88 & \\
\hline \multirow{2}{*}{\multicolumn{2}{|c|}{$\begin{array}{l}\text { Días a la semana que } \\
\text { dedica al cuidado del } \\
\text { paciente }\end{array}$}} & $>5$ días & 68 & 55.28 & 44 & 35.77 & \multirow[b]{2}{*}{1.67} & 075 & \multirow[b]{2}{*}{$0.198^{*}$} \\
\hline & & 1 a 5 días & 4 & 3.25 & 7 & 5.69 & & 3.70 & \\
\hline \multirow{2}{*}{\multicolumn{2}{|c|}{$\begin{array}{l}\text { Horas diarias } \\
\text { dedicadas al cuidado } \\
\text { del paciente }\end{array}$}} & $>8$ horas & 35 & 28.46 & 18 & 14.63 & \multirow[b]{2}{*}{1.24} & & \multirow[b]{2}{*}{0.142} \\
\hline & & 0 a 8 horas & 37 & 30.08 & 33 & 26.83 & & 1.67 & \\
\hline \multicolumn{2}{|c|}{$\begin{array}{l}\text { Antecedentes } \\
\text { Patológicos }\end{array}$} & Si & 28 & 22.76 & 17 & 13.82 & \multirow[b]{2}{*}{1.10} & $0.81-$ & \multirow[b]{2}{*}{0.529} \\
\hline $\begin{array}{l}\text { Patológic } \\
\text { Personal }\end{array}$ & s & No & 44 & 35.77 & 34 & 27.64 & & & \\
\hline \multirow{2}{*}{\multicolumn{2}{|c|}{ Dependencia }} & IS/IM & 49 & 39.84 & 8 & 6.50 & \multirow{2}{*}{2.46} & $1.74-$ & \multirow{2}{*}{0.000} \\
\hline & & Al o IL & 23 & 18.70 & 43 & 34.96 & & 3.18 & \\
\hline \multirow{2}{*}{\multicolumn{2}{|c|}{$\begin{array}{l}\text { Funcionalidad } \\
\text { Familiar }\end{array}$}} & Disfuncional & 64 & 52.03 & 39 & 31.71 & \multirow{2}{*}{1.55} & 0.89 & \multirow{2}{*}{0.066} \\
\hline & & Funcional & 8 & 6.50 & 12 & 9.76 & & 2.71 & \\
\hline \multirow{8}{*}{$\begin{array}{l}\text { Tipología } \\
\text { Familiar }\end{array}$} & Parentecro & M/ME/NP & 51 & 41.46 & 35 & 28.46 & \multirow{2}{*}{1.04} & $0.75-$ & \multirow{2}{*}{0.793} \\
\hline & & N/NE/E & 21 & 17.07 & 16 & 13.01 & & 1.45 & \\
\hline & \multirow{2}{*}{ Demografía } & Rural & 37 & 30.08 & 27 & 21.95 & 007 & $0.72-$ & \\
\hline & & Urbano & 35 & 28.46 & 24 & 19.51 & 0.0 & 1.31 & 0.000 \\
\hline & & Moderna & 22 & 17.89 & 14 & 11.38 & & $0.77-$ & \\
\hline & & Tradicional & 50 & 40.65 & 37 & 30.08 & & 1.46 & \\
\hline & $\begin{array}{l}\text { Ocupación } \\
\text { del jefe del }\end{array}$ & $\begin{array}{l}\text { No } \\
\text { empleado }\end{array}$ & 13 & 10.6 & 7 & 5.7 & 1.38 & $0.51-$ & 0.521 \\
\hline & hogar & Empleado & 59 & 48 & 44 & 35.8 & & 0.10 & \\
\hline
\end{tabular}

Fisher*

Elaborado por: los autores. Fuente: base de datos.

IS: Incapacidad severa: IM: Incapacidad moderada; IL: incapacidad leve; Al: ausencia de incapacidad; SD: severa disfuncionalidad; D: disfuncional; MF: moderadamente funcional; F: funcional. NS: nuclear simple; $\mathrm{NN}$ : nuclear numerosa; M: monoparental; ME: monoparental extendida; E: extensa; NP: no parental. 


\section{DISCUSION}

El envejecimiento de la población es uno de los más importantes fenómenos del siglo XXI, puesto que trae profundas modificaciones en las estructuras sociales, económicas y culturales de los países, situación ya experimentada en los países desarrollados. Los cambios propios del envejecimiento asociados a estilos de vida y generalmente condiciones socioeconómicas deficientes, llevan al anciano a una situación de mayor vulnerabilidad, llegando a producirse pérdida de autonomía en diferentes grados, dificultando sus actividades de la vida diaria [18].

El perfil del cuidador que se expone en el presente estudio corresponde al grupo etario comprendido entre 40 a 59 años, género femenino, baja escolaridad (primaria incompleta), casados y por lo general son los hijos del paciente a cuidar, estos hallazgos son similares a los expuestos en estudios realizados en México por Prieto y cols. [19] Palomino y cols. [20].

Se expuso también que el mayor porcentaje de los individuos se encontró sometido a sobrecarga, al igual que el análisis realizado por Costa y cols. en el 2015, esto puede deberse al tipo de paciente que se está cuidando, puede que en su mayoría tengan comorbilidades que hagan pesado su cuidado [21].

De igual manera se observó que las mujeres predominaron en el nivel de carga, en un estudio realizado en Chile por Villarroel y cols. en el 2009 encontraron que las variables de existencia de hijos y realización de una actividad laboral, además del cuidado, influyen en la presencia de sobrecarga en los cuidadores informales de adultos mayores dependientes [22], este punto también puede estar explicado porque el número de encuestados del sexo femenino fue tres veces mayor que el sexo masculino.

A medida que aumenta la carga de trabajo de la persona cuidadora, también se incrementa el riesgo de deterioro de su salud y su calidad de vida. Estas personas son descritas habitualmente como segundas víctimas de la enfermedad debido a que el problema de la dependencia no solo afecta a quien la padece, sino también a quienes tienen que facilitar las ayudas necesarias para que pueda seguir viviendo dignamente [23].
Un estudio realizado por Retamal y cols. en 2015 en Chile en adultos mayores con dependencia severa se expuso una prevalencia alta de sobrecarga [24], similar a lo expuesto en este trabajo donde la dependencia con incapacidad severa se comportó como un factor de riesgo para el desarrollo de sobrecarga.

El tiempo como cuidador es fundamental al momento de la evaluación del individuo ya que se ha reportado en diferentes estudios que es un factor determinante del síndrome de sobrecarga del cuidador, como lo fue en el caso de Flores y cols. en el 2012 [25], esto se encuentra sustentado ya que al pasar más tiempo con esta ocupación, el deterioro será progresivo, en el presente análisis se expuso al tiempo mayor de 5 años como un factor de riesgo para presentar sobrecarga, sin embargo existe aún controversia con respecto a estos resultados ya que en otras poblaciones no se ha comportado como un factor de riesgo siendo este el caso del análisis de Cardona y cols. en el 2015 [18].

\section{CONCLUSIONES}

De los 123 cuidadores fueron en su mayoría mujeres en edad comprendida entre 40 a 59 años, estaban casadas, cursaron con la instrucción primaria y en la relación familiar son hijas del adulto mayor.

Existió una alta prevalencia del síndrome de sobrecarga con el $58.50 \%$ y se encontró asociado a tener más de 5 años como cuidador y a la dependencia con incapacidad moderada a severa del paciente.

\section{INFORMACIÓN DE LOS AUTORES}

- Chamba Ortiz Paola Alexandra. Especialista en Medicina Familiar y Comunitaria. Libre ejercicio. ORCID: https://orcid.org/0000-0001-5958-4141

- Ojeda Orellana Karina Paola. Especialista en Medicina Interna. Facultad de Ciencias Médicas. Universidad de Cuenca.

ORCID: https://orcid.org/0000-0002-6695-8970

\section{CONTRIBUCIÓN DE LOS AUTORES}

CHOPA.: Planteamiento y justificación del tema, recolección de la información, tabulación de datos, presentación de resultados y conclusiones.

OOKP.: Análisis metodológico y sugerencias de presentación. 


\section{CONFLICTO DE INTERESES}

Los autores declaran no tener conflicto de intereses en la presente investigación.

\section{FUENTES DE FINANCIAMIENTO}

Autofinanciado

\section{REFERENCIAS BIBLIOGRÁFICAS}

1. Willemse E, Anthierens S, Farfan-Portet MI, Schmitz O, Macq J, Bastiaens H, et al. Do informal caregivers for elderly in the community use support measures? A qualitative study in five European countries. BMC Health Services Research [Internet]. 2016;16. Disponible en: https://www.ncbi. nlm.nih.gov/pmc/articles/PMC4947246/

2. Rowe JW, Fried L, Jackson J, Novelli W, Stone R. A Vital Direction for Health and Health Care. Discusion paper. 2016;9.

3. Brigola AG, Luchesi BM, Rossetti ES, Mioshi E, Inouye K, Pavarini SCl. Health profile of family caregivers of the elderly and its association with variables of care: a rural study. Revista Brasileira de Geriatria e Gerontologia [Internet]. mayo de 2017;20(3):409-20. Disponible en: http://www.scielo.br/scielo.php?script=sci_ arttext\&pid=S1809-98232017000300409\&In $g=e n \& t$ lng=en

4. Ampalam P, Gunturu S, Padma V. A comparative study of caregiver burden in psychiatric illness and chronic medical illness. Indian J Psychiatry [Internet]. 2012 [citado 23 de noviembre de 2018];54(3):23943. Disponible en: https://www.ncbi.nlm.nih. gov/pmc/articles/PMC3512360/

5. Yu Y, Liu Z, Tang B, Zhao M, Liu X, Xiao S. Reported family burden of schizophrenia patients in rural China. PLOS ONE [Internet]. 19 de junio de 2017 [citado 23 de noviembre de 2018];12(6):e0179425. Disponible en: https://journals.plos.org/plosone/ article id $=10.1371$ /journal . pone .0179425

6. Shamsaei F, Cheraghi F, Bashirian S. Burden on Family Caregivers Caring for Patients with
Schizophrenia. Iran J Psychiatry [Internet]. septiembre de 2015 [citado 23 de noviembre de 2018];10(4):239-45. Disponible en: https://www.ncbi.nlm.nih.gov/pmc/articles/ PMC4801494/

7. Dos Santos GD, Forlenza OV, Ladeira RB, Aprahamian I, Almeida JG, Lafer B, et al. Caregiver burden in older adults with bipolar disorder: relationship to functionality and neuropsychiatric symptoms. Psychogeriatrics. septiembre de 2017;17(5):317-23.

8. Parabiaghi A, Lasalvia A, Bonetto C, Cristofalo D, Marrella G, Tansella M, et al. Predictors of changes in caregiving burden in people with schizophrenia: a 3-year follow-up study in a community mental health service. Acta Psychiatr Scand Suppl. 2007;(437):6676.

9. Chien WT, Chan SW, Morrissey J. The perceived burden among Chinese family caregivers of people with schizophrenia. J Clin Nurs [Internet]. junio de 2007 [citado 23 de noviembre de 2018];16(6):1151-61. Disponible en: http://europepmc.org/abstract/ med/17518890

10. Hsiao C-Y, Tsai Y-F. Factors of caregiver burden and family functioning among Taiwanese family caregivers living with schizophrenia. J Clin Nurs. junio de 2015;24(11-12):1546-56.

11. Yazici E, Karabulut Ü, Yildiz M, Baskan Tekeş S, Inan E, Çakir U, et al. Burden on Caregivers of Patients with Schizophrenia and Related Factors. Noro Psikiyatr Ars [Internet]. junio de 2016;53(2):96-101. Disponible en: https://www.ncbi.nlm.nih.gov/ pmc/articles/PMC5353031/

12. Awad AG, Voruganti LNP. The burden of schizophrenia on caregivers: a review. Pharmacoeconomics. 2008;26(2):149-62.

13. Batista CF, Bandeira M, Oliveira DR. Fatores associados à sobrecarga subjetiva de homens e mulheres cuidadores de pacientes psiquiátricos. Ciência \&amp; Saúde Coletiva [Internet]. septiembre de 2015 [citado 23 de noviembre de 2018];20(9):2857-66. 
Disponible en: http://www.scielo.br/scielo. php?script=sci_abstract \&pid=S1413$81232015000902857 \&$ Ing=en\&nrm=iso\&tlng $=p t$

14. INEC. Compendio estadistico INEC [Internet]. 2014 [citado 10 de abril de 2018]. Disponible en: http://www.ecuadorencifras. gob.ec/documentos/web-inec/Bibliotecas/ Compendio/Compendio-2014/compendio_ estadistico_2014.pdf

15. Silva CAM, Orellana PAR, Nassr GNM. Valoración del estado funcional de adultos mayores con dependencia moderada y severa pertenecientes a un centro de salud familiar. Fisioter Pesq. 2015;1(1):76-83.

16. Álvarez L, González AM, Muñoz $P$. El cuestionario de sobrecarga del cuidador de Zarit: Cómo administrarlo e interpretarlo. Gaceta Sanitaria [Internet]. diciembre de 2008;22(6):618-9. Disponible en: http://scielo.isciii.es/scielo. php?script=sci_abstract \& pid=S0213$91112008000600020 \&$ Ing $=e s \& n r m=i s o \& t \operatorname{lng}$ =es

17. Gómez MTO, Torres MAP, Ortiz EP. Diseño de Intervención en Funcionamiento Familiar. Psicología para América Latina. 2009;1(1):18.

18. Cardona-Arango D, Segura Cardona ÁM, Berbesí Fernández DY, Ordoñez Molina J, Agudelo Martínez A. Características demográficas y sociales del cuidador en adultos mayores. 2011; Disponible en: http://bdigital.ces.edu.co:8080/repositorio/ handle/10946/3964

19. Prieto-Miranda SE, Arias-Ponce N, Villanueva-Muñoz EY, Jiménez-Bernardino CA. Síndrome de sobrecarga del cuidador en familiares de pacientes geriátricos atendidos en un hospital de segundo nivel [Internet]. 2015 [citado 23 de noviembre de 2018]. Disponible en: http://www.medigraphic.com/ pdfs/medintmex/mim-2015/mim156d.pdf

20. Palomino GL, Avilés AGP, Loyola LAB. Perfil del cuidador: sobrecarga y apoyo familiar e institucional del cuidador primario en el primer nivel de atención. Revista de Especialidades Médico-Quirúrgicas 2008; 13 (4):159-66.

21. Mendoza-Suárez G. Síndrome de sobrecarga en familiares encargados del cuidado de pacientes con enfermedad neurológica crónica. Rev Soc Peru Med Interna 2014;27 (1)12-18.

22. Lavoz EE, Villarroel VM, Jaque RL, Caamaño PR. Factores asociados al nivel de sobrecarga de los cuidadores informales de adultos mayores dependientes, en control en el consultorio "José Durán Trujillo", San Carlos, Chile. 2009;18:12.

23. García-Calvente $\mathrm{M}$ del $\mathrm{M}$, Mateo-Rodríguez I, Maroto-Navarro G. El impacto de cuidar en la salud y la calidad de vida de las mujeres. Gaceta Sanitaria [Internet]. 2004;18(5):8392. Disponible en: http://scielo.isciii.es/ scielo.php?script=sci_abstract \&pid=S0213$91112004000500011 \&$ Ing=es\&nrm=iso\&tlng =es

24. Retamal-Matus $\mathrm{H}$, Aguilar L, Aguilar $\mathrm{M}$, González R, Valenzano S. Factores que influyen en la sobrecarga de los cuidadores de adultos mayores en dos instituciones de la ciudad de Punta Arenas, Psicogeriatría 2015; 5 (3): 119-124.

25. Flores G E, Rivas R E, Seguel P F. Nivel de sobrecarga en el desempeño del rol del cuidador familiar de adulto mayor con dependencia severa. Ciencia y enfermería [Internet]. 2012;18(1):29-41. Disponible en: https://scielo.conicyt.cl/ scielo.php?script=sci_abstract $\&$ pid=S071795532012000100004\&lng=es\&nrm=iso\&tlng =es 